\title{
The pulsational properties of ultra-massive DB white dwarfs with carbon-oxygen cores coming from single-star evolution
}

\author{
Alejandro H. Córsico ${ }^{1,2}$, Leandro G. Althaus ${ }^{1,2}$, Pilar Gil Pons ${ }^{3}$, and Santiago Torres ${ }^{3,4}$ \\ ${ }^{1}$ Grupo de Evolución Estelar y Pulsaciones. Facultad de Ciencias Astronómicas y Geofísicas, Universidad Nacional de La Plata, \\ Paseo del Bosque s/n, 1900 La Plata, Argentina \\ e-mail: acorsico@fcaglp.unlp.edu.ar \\ 2 CCT - CONICET, La Plata, Argentina \\ 3 Departament de Física, Universitat Politècnica de Catalunya, c/Esteve Terrades 5, 08860 Castelldefels, Spain \\ 4 Institute for Space Studies of Catalonia, c/Gran Capita 2-4, Edif. Nexus 104, 08034 Barcelona, Spain
}

Received 26 November 2020 / Accepted 20 December 2020

\begin{abstract}
Context. Ultra-massive white dwarfs are relevant for many reasons: their role as type Ia supernova progenitors, the occurrence of physical processes in the asymptotic giant branch phase, the existence of high-field magnetic white dwarfs, and the occurrence of double white dwarf mergers. Some hydrogen-rich ultra-massive white dwarfs are pulsating stars and, as such, they offer the possibility of studying their interiors through asteroseismology. On the other hand, pulsating helium-rich ultra-massive white dwarfs could be even more attractive objects for asteroseismology if they were found, as they should be hotter and less crystallized than pulsating hydrogen-rich white dwarfs, something that would pave the way for probing their deep interiors.

Aims. We explore the pulsational properties of ultra-massive helium-rich white dwarfs with carbon-oxygen and oxygen-neon cores resulting from single stellar evolution. Our goal is to provide a theoretical basis that could eventually help to discern the core composition of ultra-massive white dwarfs and the scenario of their formation through asteroseismology, anticipating the possible future detection of pulsations in helium-rich ultra-massive white dwarfs.

Methods. We focus on three scenarios for the formation of helium-rich ultra-massive white dwarfs. First, we consider stellar models coming from two recently proposed single-star evolution scenarios for the formation of ultra-massive white dwarfs with carbonoxygen cores that involve the rotation of the degenerate core after core helium burning and reduced mass-loss rates in massive asymptotic giant branch stars. Finally, we contemplate ultra-massive oxygen-neon core white-dwarf models resulting from standard single-star evolution. We compute the adiabatic pulsation gravity-mode periods for models in a range of effective temperatures, embracing the instability strip of average-mass pulsating helium-rich white dwarfs, and we compare the characteristics of the modetrapping properties for models of different formation scenarios through the analysis of the period spacing.

Results. Given that the white dwarf models coming from the three scenarios considered are characterized by distinct core chemical profiles, we find that their pulsation properties are also different, thus leading to distinctive signatures in the period-spacing and modetrapping properties.

Conclusions. Our results indicate that in the case of an eventual detection of pulsating ultra-massive helium-rich white dwarfs, it would be possible to derive valuable information encrypted in the core of these stars in connection with the origin of such exotic objects. This is of the utmost importance regarding recent evidence for the existence of a population of ultra-massive white dwarfs with carbon-oxygen cores. There will soon be many opportunities to detect pulsations in these stars through observations collected with ongoing space missions.
\end{abstract}

Key words. stars: evolution - stars: interiors - white dwarfs - asteroseismology - stars: oscillations - dense matter

\section{Introduction}

Massive white-dwarf (WD) stars have become a topic of great interest in recent years as their origin and evolutionary properties are key to understand type Ia supernova, the occurrence of physical processes in the asymptotic giant branch (AGB) phase, the theory of crystallization and the existence of high-field magnetic WDs, as well as to help our understanding of double WD mergers (Dunlap et al. 2015; Reindl et al. 2020). The mass distribution of the entire WD population is clearly peaked at $M_{\star} \sim 0.6 M_{\odot}$, but massive WDs also show a well-defined peak at $M_{\star} \sim 0.82 M_{\odot}$ (e.g., Kleinman et al. 2013; Jiménez-Esteban et al. 2018), which has been attributed, among other factors, to the empirical crystallization delay at higher masses observed in Gaia (Tremblay et al. 2019a,b; Kilic et al. 2020). In addition, the existence of ultra-massive WDs $\left(M_{\star} \gtrsim 1 M_{\odot}\right)$ has been reported in numerous studies (Castanheira et al. 2010, 2013; Hermes et al. 2013; Kepler et al. 2016; Curd et al. 2017; Gagné et al. 2018; Hollands et al. 2020; Bédard et al. 2020).

The standard scenario for the formation of an ultra-massive WD involves single progenitor stars of initial masses higher than 6-9 $M_{\odot}$ (the precise value strongly depends on the input physics and metallicity), which experience off center carbon (C) burning during the super AGB when the carbon-oxygen (CO) core mass has grown to about $1.05 M_{\odot}$, thus eventually leading to the formation of ultra-massive WDs with oxygen-neon (ONe) cores (see, e.g., Garcia-Berro \& Iben 1994; Siess 2007; Doherty et al. 2017). An alternative scenario involves the double WD merger. Theoretical computations indicate that double WD mergers would contribute, at least to some extent, to the single 
massive WD population (Toonen et al. 2017; Maoz et al. 2018). In particular, Temmink et al. (2020) predict that a fraction of all observable single WDs more massive than $0.9 M_{\odot}$ within 100 pc might primarily result from the merger of two WDs. Also, Cheng et al. (2020) conclude that about $20 \%$ of massive and ultra-massive WDs in the mass range of $0.8-1.3 M_{\odot}$ result from double WD mergers.

Evidence from Gaia kinematics of large cooling delays of ultra-massive WDs on the "Q-branch" reported by Cheng et al. (2020) and the location of the Q-branch on the color-magnitude diagram are consistent with a population of ultra-massive WDs with CO cores (see also Tremblay et al. 2019b; Bauer et al. 2020). Given recent studies based on post-merger evolutionary calculations suggesting the formation of an ONe core after a WD merger in ultra-massive WDs (Shen et al. 2012; Schwab 2021), the existence of a population of ultra-massive WDs with $\mathrm{CO}$ cores (UMCO WDs) is difficult to understand. Recently, Althaus et al. (2021) have explored single-evolution scenarios which could lead to the formation of UMCO WDs. These authors have studied the evolutionary and pulsational properties of the resulting DA WDs and compared them with those of ultra-massive WDs with ONe cores, thus establishing a theoretical basis that could eventually help to infer the core composition of ultra-massive WDs and the scenario of their formation. Specifically, Althaus et al. (2021) study two possible single evolution scenarios for the formation of UMCO WDs. One scenario exploits wind rates and convective boundary uncertainties during the thermally-pulsing AGB (TP-AGB) phase, and it involves the reduction of these rates below the values given by standard prescriptions (see, e.g., Decin et al. 2019). The other scenario requires the occurrence of rotation in degenerate $\mathrm{CO}$ cores, which is naturally expected as a consequence of core contraction at the end of core helium (He) exhaustion (Dominguez et al. 1996). Althaus et al. (2021) show that both the evolutionary and pulsational properties of the UMCO WDs that formed through these two single evolution scenarios are markedly different from those of ultra-massive WDs with ONe cores (UMONe WDs). Such differences in evolutionary and pulsational properties may eventually be used to shed light on the core composition of ultra-massive WDs.

The chemical stratification and internal structure of pulsating WDs and pulsating ultra-massive WDs, in particular, can be probed by means of asteroseismology in principle (Winget \& Kepler 2008; Fontaine \& Brassard 2008; Althaus et al. 2010; Córsico et al. 2019a). Indeed, several ultra-massive H-rich WDs (DA WDs) exhibit $g$ (gravity)-mode pulsational instabilities (Kanaan et al. 2005; Castanheira et al. 2010, 2013; Hermes et al. 2013; Curd et al. 2017; Rowan et al. 2019), and they are part of the ZZ Ceti (or DAV) class of variable H-rich WDs. A recent attempt to explore the internal structure of ultra-massive ZZ Cetis stars via asteroseismology was conducted by Córsico et al. (2019b). These authors emphasize the need for the detection of more periods and more pulsating ultra-massive WDs, something that could be achieved soon with observations from space, such as those of the Transiting Exoplanet Survey Satellite (TESS; Ricker et al. 2015).

To explore the internal structure of pulsating WDs through asteroseismology, it is crucial to employ stellar models with detailed chemical profiles resulting from the complete evolution of their progenitor stars, as well as time-dependent element diffusion during the WD evolution. Indeed, the details pertaining to the shape of the internal chemical profiles of WDs are key in relation to the characteristics of the $g$-mode pulsation spectrum of these stars and, in particular, to the mode trapping properties.
This was realized almost two decades ago by the La Plata Group ${ }^{1}$ in the case of DAV stars (e.g., Córsico et al. 2001), pulsating He-rich atmosphere WD stars, called DBV or V777 Her stars (e.g., Althaus \& Córsico 2004), and pulsating PG 1159 stars, also called GW Vir stars (e.g., Córsico \& Althaus 2006).

In this paper we extend the scope of the study of Althaus et al. (2021) by exploring the pulsational properties of the ultramassive WDs with He-rich atmospheres (DB WDs) resulting from single-star evolution. In the case of H-deficient WDs, the mass distribution shows an apparent deficiency of ultra-massive objects (Tremblay et al. 2019a; Kepler et al. 2019). Despite the fact that ultra-massive H-deficient WDs are not as common as ultra-massive H-rich WDs, several pieces of observational evidence are found. Indeed, some recent works point to the existence of a small subpopulation of ultra-massive DO (He-rich atmospheres with ionization lines) and DB WDs (Reindl et al. 2014; Bédard et al. 2020). Another class of H-deficient ultramassive WDs are the DQ WDs, which show He and $\mathrm{C}$ at their atmospheres (Kleinman et al. 2013; Koester et al. 2020). In particular, Reindl et al. (2014) found one ultra-massive DO WD from the data release 10 (DR10) of the Sloan Digital Sky Survey (SDSS; York et al. 2000) with a mass of $1.07 M_{\odot}$ if it is an $\mathrm{ONe}$-core $\mathrm{WD}$, or $1.09 M_{\odot}$ if it is a CO-core WD. On the other hand, Bédard et al. (2020) find five ultra-massive DO WDs stars $^{2}$ from DR12 of the SDSS, with masses in the range of $1.01 \leq M_{\star} / M_{\odot} \leq 1.06$. Richer et al. (2019) have reported the existence of an ultra-massive DB WD with no $\mathrm{H}$ lines in a young open cluster. Its effective temperature, which is in excess of $25000 \mathrm{~K}$, places this ultra-massive DB WD inside the DBV instability strip. Recently, Pshirkov et al. (2020) discovered an ultra-massive WD with He-rich atmosphere and traces of $\mathrm{H}$ (DBA spectral class) with $T_{\text {eff }}=31200 \pm 1200 \mathrm{~K}$ and $M_{\star}=1.33 M_{\odot}$ exhibiting photometric variability with a single period of $353.456 \mathrm{~s}$, which could be due to fast rotation, supporting a merger scenario for its formation. However, it cannot be completely ruled out that the variability is due to pulsations since it is close to the blue edge of the DBV instability strip. We note that a significant number of ultra-massive He-atmosphere WDs are magnetic, including the one in Richer et al. (2019) and also the hot DQ WDs (see, e.g., Dufour et al. 2013). The presence of a strong magnetic field could complicate the study of pulsations since an intense magnetic field would be capable with inhibiting convection and, therefore, could have a dramatic effect on the driving mechanism of the pulsations (Tremblay et al. 2015).

In this paper, we show that pulsational properties of the ultramassive DB WDs are much more strongly dependent on their formation scenario than in the case of the DA WD ones studied in Althaus et al. (2021). This is due to the fact that pulsating ultra-massive DB WDs, if they exist, must be much hotter than ultra-massive ZZ Cetis and, therefore, their cores must be less crystallized. As a result, $g$-mode pulsations can penetrate much deeper into the star, thus carrying valuable information about the core chemical structure and composition. Hence, the potential detection of pulsating ultra-massive DB WDs would constitute a clear and unique opportunity to discern the core composition and origin of the ultra-massive WD population in general.

The paper is organized as follows. In Sect. 2 we describe the stellar codes employed to compute the evolutionary and pulsational properties of our DB WD models, and we briefly

\section{1 http://fcaglp.unlp.edu.ar/evolgroup}

2 They consist of two DO, two DOZ (atmospheres with traces of metals), and one DOA (atmospheres with $\mathrm{H}$ lines) WDs (Bédard et al. 2020). 
summarize the single stellar evolution scenarios that lead to our initial UMCO WD models. In Sect. 3 we describe the pulsational properties of the resulting WDs. Finally, in Sect. 4 we summarize the main findings of the paper.

\section{Numerical codes and ultra-massive WD models}

The evolutionary and pulsational properties of our UMCO and UMONe DB WD models were computed with the same stellar codes we used in Althaus et al. (2021). For the pulsational analysis, we used the LP-PUL pulsation code described in Córsico \& Althaus (2006). The code adopts the "hard-sphere" boundary conditions to account for the effects of crystallization on the pulsation spectrum of $g$ modes. These conditions assume that the amplitude of the eigenfunctions of $g$-modes is null below the solid and liquid interface because of the non-shear modulus of the solid, as compared with the fluid region (see Montgomery \& Winget 1999). The inner boundary condition for pulsations corresponds to the mesh-point at the crystallization front (see Córsico et al. 2004, 2005, 2019b; De Gerónimo et al. 2019). The Brunt-Väisälä frequency was computed as in Tassoul et al. (1990). The computation of the Ledoux term $B$ includes the effects of having multiple chemical species that vary in abundance. For the computation of the WD evolutionary models, we used the LPCODE stellar evolutionary code which has been widely used and tested in numerous stellar evolutionary contexts of low-mass and WD stars (see Althaus et al. 2003, 2005, 2015; Salaris et al. 2013; Miller Bertolami 2016; Silva Aguirre et al. 2020; Christensen-Dalsgaard et al. 2020, for details). In particular, the treatment of crystallization was based on phase diagrams of Horowitz et al. (2010) for dense CO mixtures, and that of Medin \& Cumming (2010) for ONe mixtures. LPCODE considers a new full-implicit treatment of time-dependent element diffusion, which includes thermal and chemical diffusion and gravitational settling (Althaus et al. 2020b). In contrast to Althaus et al. (2021), we considered the effect of the Coulomb separation of ions in this work (Chang et al. 2010; Beznogov \& Yakovlev 2013; Althaus et al. 2020a). As a result of this Coulomb diffusion, ions with larger $Z$ move to deeper layers. Coulomb diffusion is not negligible in the dense $\mathrm{C}$ - and He-rich envelopes of ultra-massive WDs, thus preventing the inward diffusion of $\mathrm{He}$ toward the core and leading to a much sharper transition region.

Now, we summarize the main formation scenarios of our UMCO WD models. Full details can be found in Althaus et al. (2021). These authors explore two different single-evolution scenarios that can lead to the formation of UMCO WDs. One of them involves a reduction in the mass-loss rates which are usually adopted for the evolution of massive AGB stars. They show that, in this case, if the minimum $\mathrm{CO}$-core mass for the occurrence of C-burning is not reached before the TP-AGB phase, a UMCO WD of a mass larger than $M_{\star} \gtrsim 1.05 M_{\odot}$ can be formed as a result of the slow growth of the $\mathrm{CO}$-core mass during the TP-AGB phase. For this to occur, the mass-loss rates of massive AGB stars need to be at least 5-20 times lower than standard mass-loss rates when a search algorithm for convective neutrality is used to determine convective boundaries. Such treatment favored very efficient third dredge-up, which hampered core growth during the TP-AGB. On the other hand, when the strict Schwarzschild criterion is used for the determination of convective boundaries, core growth is faster, and the required reduction of standard mass-loss rates drops to a factor of 2 . The reduction in mass-loss rates necessary to form UMCO WDs cannot be discarded and this is in line with different pieces of recent observational evidence indicating that mass-loss rates are lower than expected from current models (e.g., Decin et al. 2019).

The other single evolution scenario that leads to the formation of UMCO WDs involves rotation of the degenerate core, which results after core He burning at the onset of the AGB phase (Dominguez et al. 1996). As these authors, Althaus et al. (2021) find that the lifting effect of rotation delays the occurrence of the second dredge-up and it maintains the maximum temperature at lower values than that required for off-center $\mathrm{C}$-ignition. Hence, $\mathrm{C}$-burning is prevented and the mass of the resulting $\mathrm{CO}$ core is larger than that at which C-burning is expected in the absence of rotation. As a result, the mass of the degenerate $\mathrm{CO}$ core is larger than $1.05 M_{\odot}$ before the TP-AGB sets in. Althaus et al. (2021) find that UMCO WDs can be formed even for very low rotation rates and that the range of initial masses leading to UMCO WDs widens as the rotation rate increases, whereas the range for the formation of ONe-core WDs decreases significantly.

Finally, we have considered UMONe WDs resulting from off-center C-burning during the single evolution of the progenitor star. Such WD models were studied in detail by Camisassa et al. (2019). The progenitors of our UMONe WDs experienced a violent $\mathrm{C}$-ignition phase followed by the development of an inward-propagating convective flame that transforms the $\mathrm{CO}$ core into a degenerate $\mathrm{ONe}$ one. In this scenario, ultra-massive WDs with stellar masses larger than $M_{\star} \gtrsim 1.05 M_{\odot}$ and composed of ${ }^{16} \mathrm{O}$ and ${ }^{20} \mathrm{Ne}$ - with traces of ${ }^{23} \mathrm{Na}$ and ${ }^{24} \mathrm{Mg}-$ are expected to emerge (Siess 2007, 2010).

Our starting ultra-massive DB WD configurations were extracted from the ultra-massive DA WDs with a stellar mass of $1.159 M_{\odot}$ studied in Althaus et al. (2021), and they formed through the three scenarios previously mentioned, to which we have simply removed the whole $\mathrm{H}$ content at the beginning of the WD cooling track. The evolution of the resulting ultra-massive DB WD configurations was followed down to the red edge of the DBV instability strip, that is, at $T_{\text {eff }} \sim 20000 \mathrm{~K}$, in a selfconsistent way with the changes in the internal chemical distribution that result from the mixing of all the core-chemical components induced by the mean molecular weight inversion left by progenitor evolution, element diffusion, and phase separation of core-chemical constituents upon crystallization. The chemical profiles of the resulting ultra-massive DB WD configurations are shown in Fig. 1. The top panel illustrates the chemical profile for the ONe-core WD, the second and third panels depict the chemical profiles resulting from reducing the mass-loss rates of an initially $7.8 M_{\odot}$ progenitor and from considering core rotation in the AGB phase of an initially 7.6 $M_{\odot}$ progenitor, respectively. The chemical profiles correspond to ultra-massive DB WD models at the onset of their cooling phase prior to the onset of element diffusion and after the core mixing induced by the inversion of the mean molecular weight. The chemical structure of both the core and the envelope of the resulting ultra-massive WDs strongly depends on the evolutionary scenario that leads to their formation. In particular, because of the lower temperature and pressure prompted by core rotation favoring the formation of a less degenerate core, the He content of the UMCO WD resulting from rotation is larger than for the UMCO WD resulting from reduced mass-loss rates and the UMONe WD (Althaus et al. 2021).

During WD cooling, the internal chemical distribution of our models mainly changes due to element diffusion and as a result of phase separation of the core chemical constituents upon crystallization. By the time evolution has proceeded to the blue (hot) edge of DBV instability strip, that is, at $T_{\text {eff }} \sim 30000 \mathrm{~K}$, chemical diffusion has strongly smoothed out the rather abrupt 

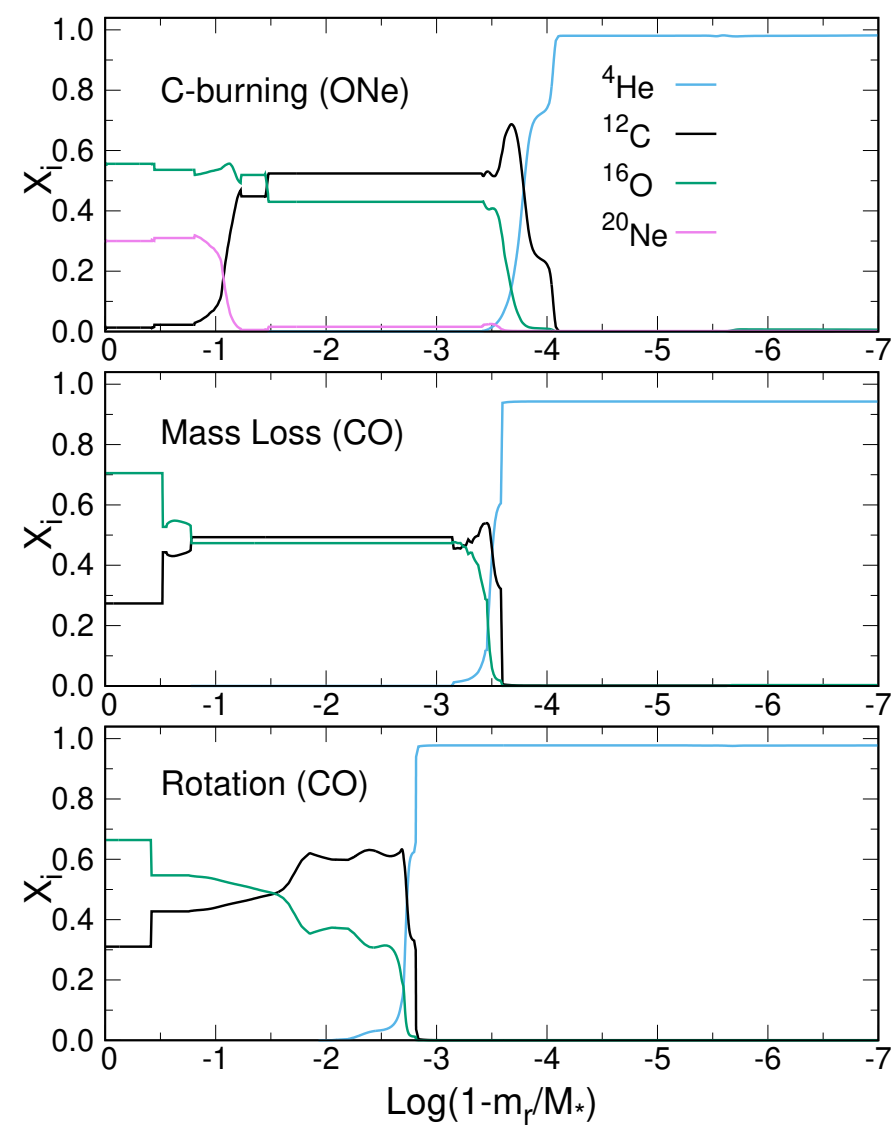

Fig. 1. Abundance by mass of ${ }^{4} \mathrm{He},{ }^{12} \mathrm{C},{ }^{16} \mathrm{O}$, and ${ }^{20} \mathrm{Ne}$ versus the outer ass coordinate for the $1.156 M_{\odot}$ DB WD models resulting from the evolutionary scenarios we studied. From top to bottom: ONe WD model from Camisassa et al. (2019), CO WD model resulting from reduced mass loss during progenitor evolution, and CO WD model implied by rotation.

initial He/C transition of our WD models (see Fig. 2). Figure 3 shows the chemical profiles at the red (cool) edge of DBV instability strip, that is, at $T_{\text {eff }} \sim 20000 \mathrm{~K}$. As evolution proceeds along the instability strip, element diffusion barely modifies the chemical profiles. For the stellar mass value we considered, only the UMONe WD sequence develops core crystallization, while the WD model evolves along the instability strip. This is due to the larger Coulomb interactions prevailing at the $\mathrm{ONe}$ core, as compared with the $\mathrm{CO}$ core. Crystallization for this sequence starts at $T_{\text {eff }} \sim 24500 \mathrm{~K}$, and by the time it abandons the instability strip, the mass of the crystallized core amounts to about $34 \%$. The shape of the chemical profile is not only modified by crystallization in the crystallized ONe core left behind, but also in liquid regions beyond the crystallization front. These changes in the core composition at the liquid regions are expected to impact the theoretical pulsational spectrum of ultra-massive WDs (see De Gerónimo et al. 2019).

In order to explore the dependence of the pulsational properties of our models of ultra-massive DB WDs with the stellar mass, we generated an additional sequence of $M_{\star}=1.29 M_{\odot}$ for each scenario by artificially scaling the mass value of each $1.156 M_{\odot}$ sequence at high luminosities. In this case, due to the higher mass, all the model sequences experienced core crystallization as they were evolving along the instability domain of DBVs. In particular, at the blue edge of the instability strip, the mass of the crystallized core amounts to about $54 \%$ in the
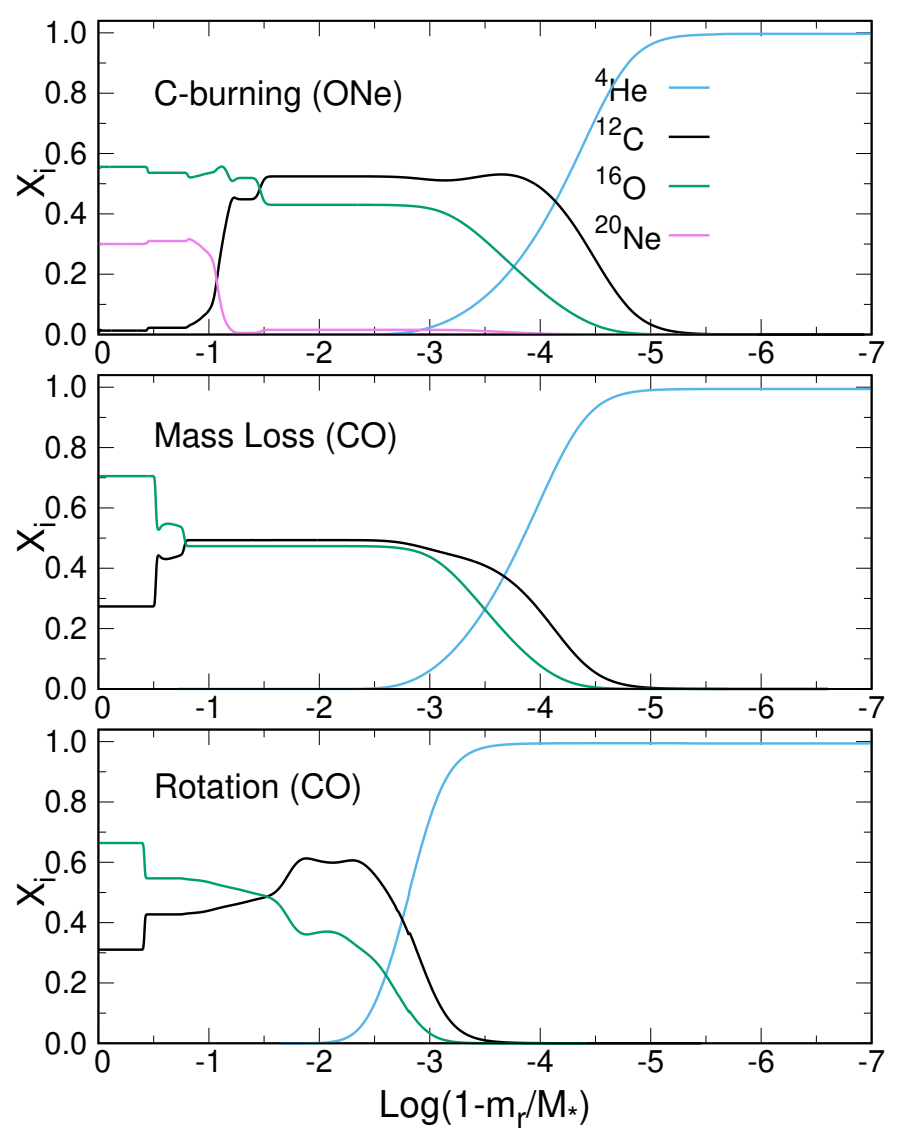

Fig. 2. Same as Fig. 1, but at an effective temperature of $T_{\text {eff }} \sim 30000 \mathrm{~K}$, close to the blue edge of the instability domain of DBV WDs. The stellar mass of the models is $M_{\star}=1.156 M_{\odot}$.

UMONe WD model, $26 \%$ for the UMCO WD model resulting from reduced mass loss, and $22 \%$ in the case of the UMCO WD model implied by rotation. These percentages change to $93 \%$, $79 \%$, and $79 \%$, respectively, by the time the models reach the red boundary of the instability strip.

\section{Pulsation results}

In this section, we compare the pulsational properties of our 1.156 $M_{\odot}$ and $1.29 M_{\odot}$ UMCO DB WD models, which are the result of two single scenarios based on rotation and reduced mass-loss rates, with those predicted for the UMONe DB WD models resulting from off-center $\mathrm{C}$-burning during the single evolution of progenitor stars (Siess 2010). For simplicity, we restrict ourselves to showing the results for two extreme temperatures: one corresponding to the hot edge, and the other being typical of the cool edge of the DBV instability strip.

In Figs. 4 and 5 we compare the logarithm of the squared Brunt-Väisälä and Lamb frequencies (see Unno et al. 1989, for their definition) in terms of the outer mass fraction of the $1.156 M_{\odot}$ DB WDs models at the blue and red edge of the DBV instability strip, respectively. The shape of the Brunt-Väisälä frequency has a strong impact on the $g$-mode period spectrum and mode-trapping properties of pulsating WDs. The BruntVäisälä frequency of our ultra-massive models does not exhibit relevant features in the outer layers. This is because chemical diffusion has strongly smoothed out the ${ }^{16} \mathrm{O}^{12} \mathrm{C}^{4} \mathrm{He}$ interface (see Figs. 2 and 3), translating into a very smooth shape of the Brunt-Väisälä frequency. However, there exist dominant features 

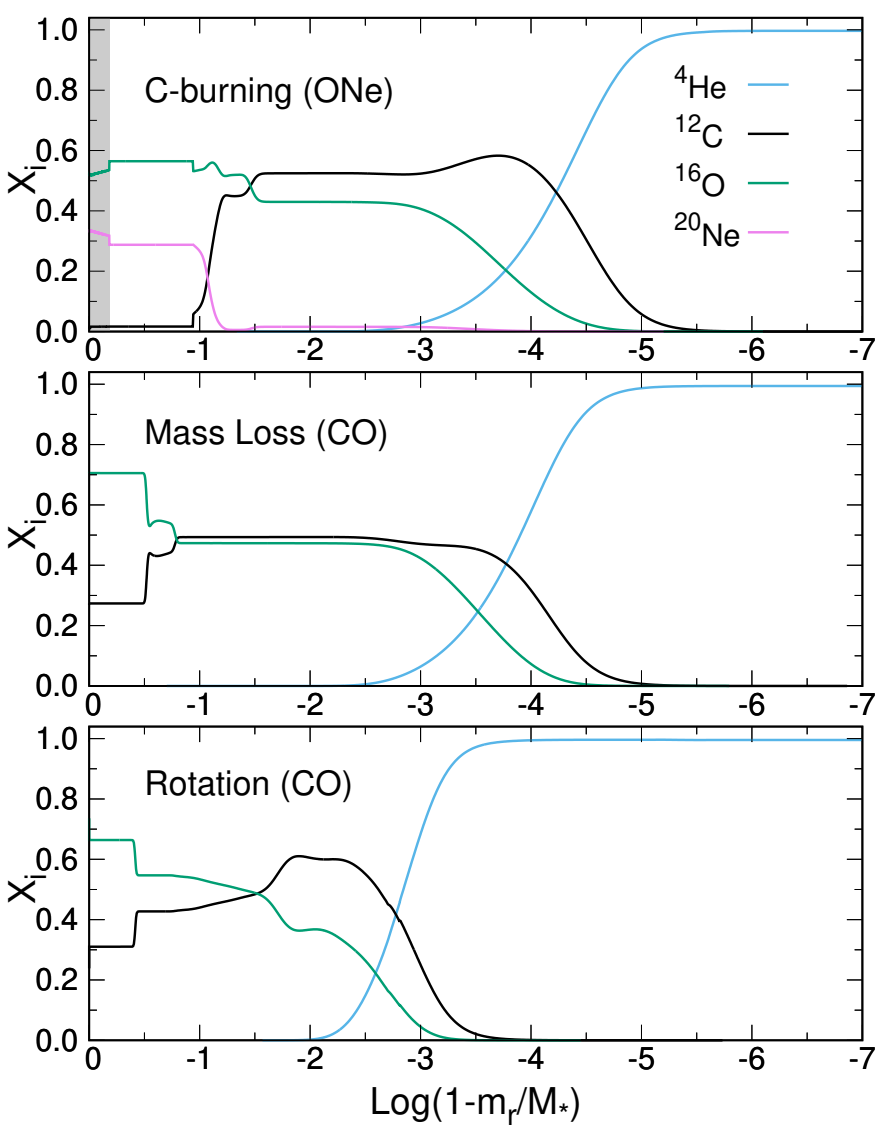

Fig. 3. Same as Fig. 2, but at an effective temperature of $T_{\text {eff }} \sim 20000 \mathrm{~K}$, close to the red edge of the instability domain of DBV WDs. The gray area in the upper panel indicates the domain of core crystallization. The percentage of crystallized mass is $\sim 34 \%$.

in the run of the Brunt-Väisälä frequency associated with the innermost chemical transition regions. We note that different bumps are present at different locations, reflecting the location of the core chemical transitions in each model.

In Figs. 6 and 7, we show the $\Delta \Pi-\Pi$ diagram, that is to say the separation of periods having a consecutive radial order $k$ (the "forward period spacing" $\Delta \Pi \equiv \Pi_{k+1}-\Pi_{k}$ ) versus the periods of $\ell=1$ pulsation $g$ modes, for our $1.156 M_{\odot}$ DB WDs models at the blue $\left(T_{\text {eff }} \sim 30000 \mathrm{~K}\right)$ and red $\left(T_{\text {eff }} \sim 20000 \mathrm{~K}\right)$ edge of the DBV instability strip, respectively. Such diagrams constitute a sensitive tool for studying the mode-trapping properties in pulsating WDs. Mode-trapping features, which are inflicted by bumps in the Brunt-Väisälä frequency, manifest themselves in that at a given $T_{\text {eff }}$ value, the separation between consecutive periods departs from the mean (constant) period spacing. For the stellar mass considered and for the complete range of effective temperatures of the instability strip, the models exhibit notable mode-trapping features for the whole period range. The $\Delta \Pi$ value is characterized by maxima and minima, typical of WD models harboring one or more chemical interfaces. These maxima and minima represent departures from a constant period separation, which is represented in the figures by the asymptotic period spacing (horizontal black-dotted line).

The $1.156 M_{\odot}$ UMCO DB WD models we analyze here do not develop crystallization during the DBV instability strip, allowing the pulsation modes to "feel" the presence of the chemical transition regions in the core, and eventually producing the mode-trapping features in all of the models. This is in con-
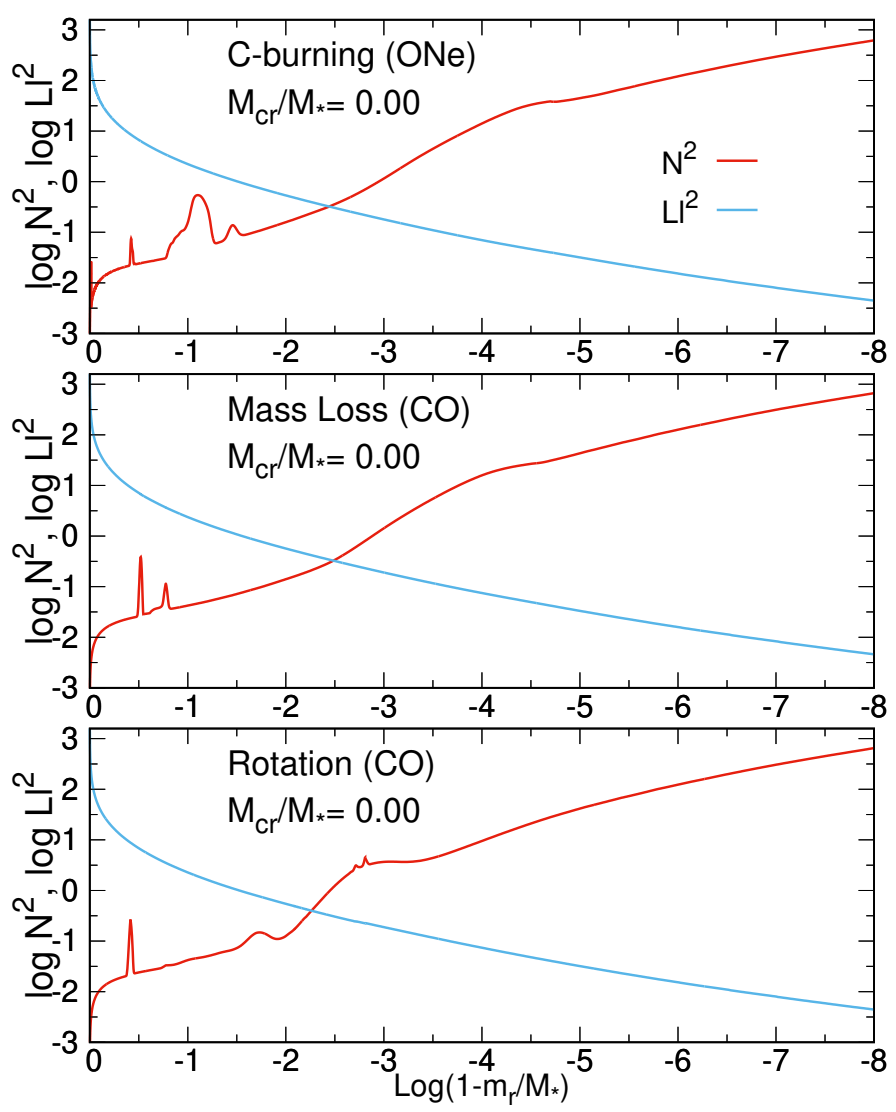

Fig. 4. Logarithm of the squared Brunt-Väisälä and Lamb frequencies (red and blue lines, respectively) corresponding to the same models analyzed in Fig. 2, characterized by $M_{\star}=1.156 M_{\odot}$ and $T_{\text {eff }} \sim 30000 \mathrm{~K}$. The Lamb frequency corresponds to dipole $(\ell=1)$ modes.

trast to the situation encountered in the ultra-massive DA WDs, which, at the evolutionary stages they pulsate, have their cores mostly crystallized. In the presence of crystallization, any chemical interface located within the crystallized region in each model has no relevance to the pulsation properties of the $g$ modes, since their eigenfunctions cannot penetrate the solid region of the stellar core. In the case of the $1.156 M_{\odot}$ UMCO DB WDs models studied here, the pulsations are able to probe from the surface to the very center of the star all along the instability strip. A notable feature of Fig. 6 is that the pattern of forward period spacing for the models with a $\mathrm{CO}$ core is quite different from that of the ONe-core WD model, particularly for modes with periods longer than $\sim 200 \mathrm{~s}$. So, in principle, for pulsating ultra-massive DB WDs with stellar masses close to $1.16 M_{\odot}$ evolving at the hot edge of the DBV instability strip, it could be possible to distinguish the different core chemical structures and compositions through pulsations, and thus provide clues as to the evolutionary channels that led to their formation.

The global characteristics of forward period spacing are similar for the $1.156 M_{\odot}$ models associated with the three scenarios when they are close to the cool boundary of the DBV instability strip (Fig. 7). The only difference-which is barely visible-is related to the asymptotic period spacing, which slightly exceeds $\sim 30 \mathrm{~s}$ in the case of the ONe-core WD model due to the crystallized region, compared to the cases of the UMCO WD models, which are not crystallized at all and have asymptotic period spacings that are slightly less than $\sim 30 \mathrm{~s}$. In practice, the mean period spacing is very difficult to measure because a moderately high number of periods should be observed. And it is even more 

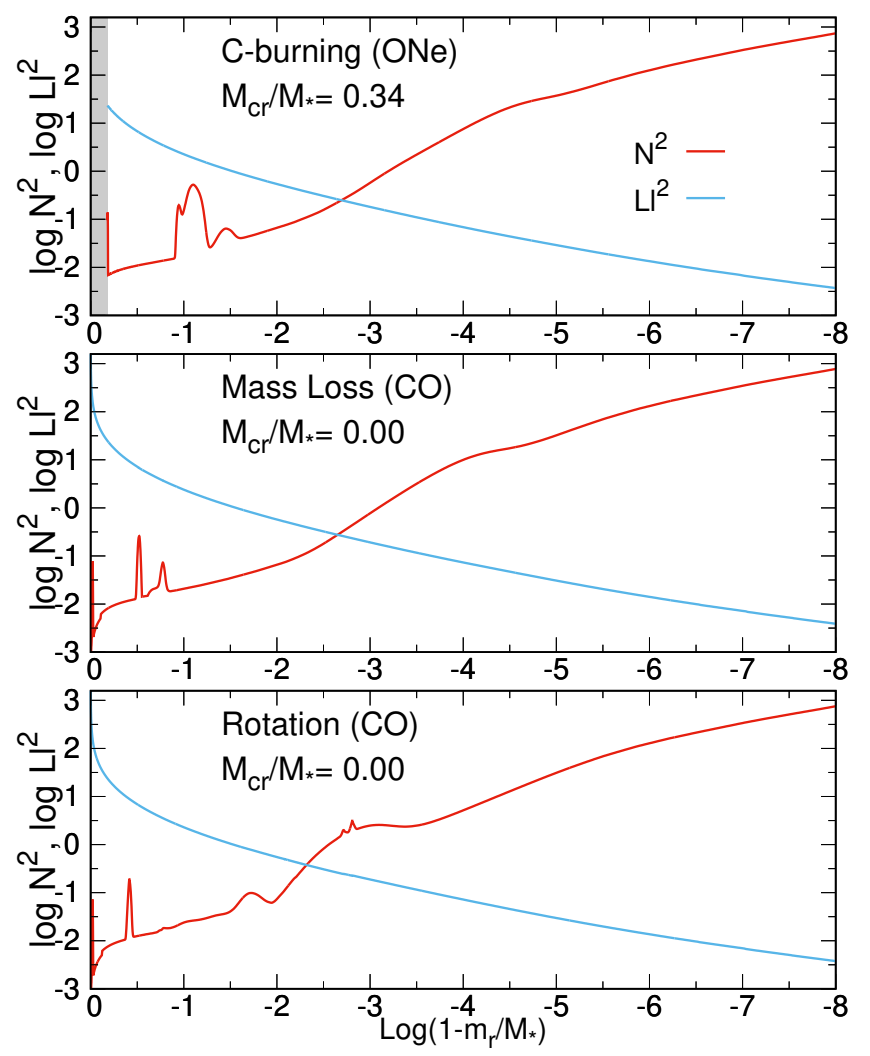

Fig. 5. Same as Fig. 4, but for the models analyzed in Fig. 3, with $M_{\star}=1.156 M_{\odot}$ and $T_{\text {eff }} \sim 20000 \mathrm{~K}$. The gray area in the upper panel corresponds to the crystallized part of the model.

difficult to observationally estimate the tiny difference between the values of the mean period spacing of one formation scenario or another, as predicted by our theoretical computations. We conclude that, in the case of pulsating ultra-massive DB WDs with masses close to $1.16 M_{\odot}$ and temperatures near $T_{\text {eff }} \sim$ $20000 \mathrm{~K}$, we would not be able to distinguish between the different types of structures and chemical compositions of the core, thus depriving the possibility of inferring their evolutionary origin.

We turn now to the case of a more massive model sequence, that of $1.29 M_{\odot}$. Because of larger central densities, crystallization develops in the core of the models for all the formation scenarios during the DBV instability strip, even at its hot boundary. In Figs. 8 and 9, we depict the forward period spacing in terms of periods for models of $M_{\star}=1.29 M_{\odot}$ at $T_{\text {eff }} \sim 30000 \mathrm{~K}$ and $T_{\text {eff }} \sim 20000 \mathrm{~K}$, respectively. As can be seen, again at the high effective temperatures typical of the blue edge of the instability strip (Fig. 8), the WD models that come from the three evolutionary scenarios all exhibit deviations from a constant period spacing due to mode trapping. We note that although the modetrapping amplitudes-the magnitude of the deviations from the constant period spacing-are similar in the three models, it is nevertheless possible to distinguish between different trapping cycles, that is to say the intervals of periods between two consecutive minima of $\Delta \Pi$ in the case of the CO-core model with rotation, in relation to the other two scenarios. This difference could be exploited, in principle, from an observational point of view to distinguish between the core chemical composition and the evolutionary channel of the star, provided that a large number of $g$ modes with consecutive $k$ values were detected in a $\sim 1.30 M_{\odot}$ DB WD star at high effective temperatures.
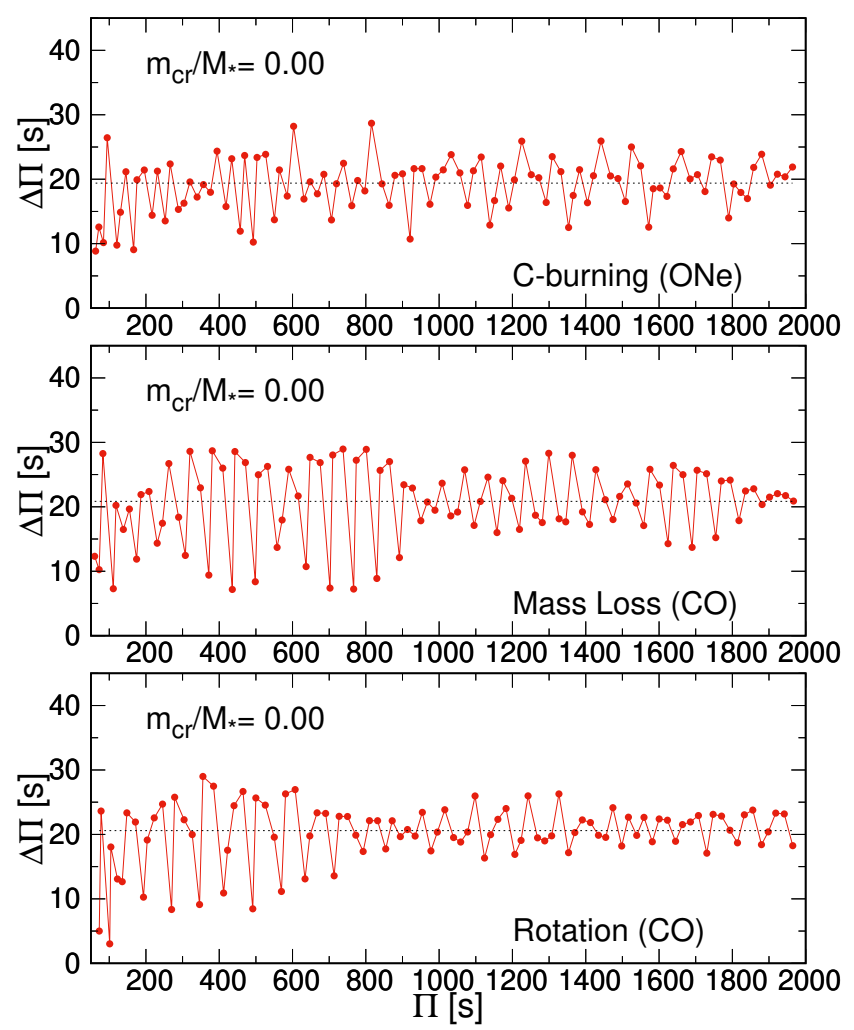

Fig. 6. Forward period spacing $(\Delta \Pi)$ in terms of the periods of $\ell=1$ pulsation $g$ modes, corresponding to the models analyzed in Figs. 2 and 4, characterized by $M_{\star}=1.156 M_{\odot}$ and $T_{\text {eff }} \sim 30000 \mathrm{~K}$. The horizontal black-dotted line is the asymptotic period spacing.

At the other end of the DBV instability strip, near the red boundary (Fig. 9), the ONe-core WD model exhibits strongly weakened mode-trapping signals in the period spacing. This behavior can be understood as follows. For the ONe-core WD model, which starts to crystallize at $T_{\text {eff }} \sim 39700 \mathrm{~K}$, the chemical interface located at $\log \left(1-m_{r} / M_{\star}\right) \sim-1$-which is responsible for the mode trapping exhibited by this WD model-ends up being contained in the crystallized part of the core by the time the model reaches the cool edge of the DBV instability strip. This can be seen in the upper panel of Fig. 10, corresponding to $T_{\text {eff }} \sim 20000 \mathrm{~K}$. This results in a very smooth BruntVäisälä frequency (see lower panel of Fig. 10), resulting in an almost constant period spacing (upper panel of Fig. 9). In contrast to this, in the case of the CO-core models, the period spacing shows notorious mode-trapping features, which are due to the presence of spikes in the Brunt-Väisälä frequency that are still within the propagation cavity of the $g$ modes (i.e., outside the crystallized regions). We conclude that in the case of very massive $\left(\sim 1.30 M_{\odot}\right)$ ONe-core pulsating DB WDs near the red edge of the DBVs instability strip, the period spacing should be almost devoid of departures from a constant period separation. This would help to distinguish ONe-core pulsating DB WDs near the red edge of the DBVs instability strip from their COcore counterparts.

\section{Summary and conclusions}

In this paper, we have extended the scope of the analysis of Althaus et al. (2021) by exploring the adiabatic pulsational properties of ultra-massive DB WDs resulting from single-star evolution. Ultra-massive H-deficient WDs are less frequent than 
A. H. Córsico et al.: Pulsational properties of ultra-massive DB white dwarfs
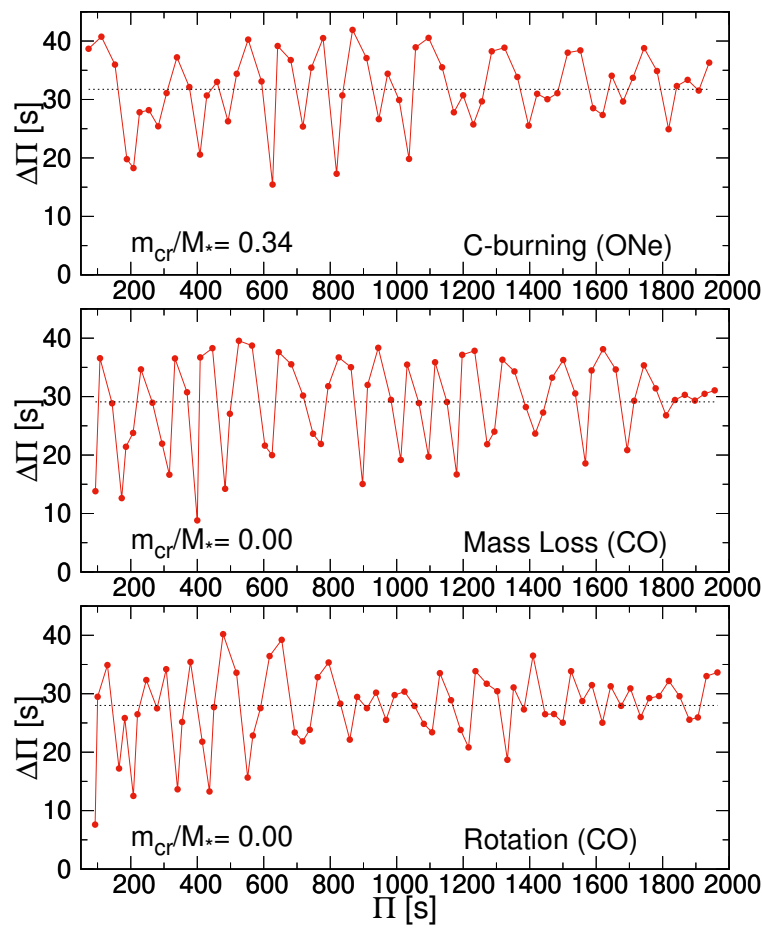

Fig. 7. Same as Fig. 6, but for the models analyzed in Figs. 3 and 5, with $M_{\star}=1.156 M_{\odot}$ and $T_{\text {eff }} \sim 20000 \mathrm{~K}$

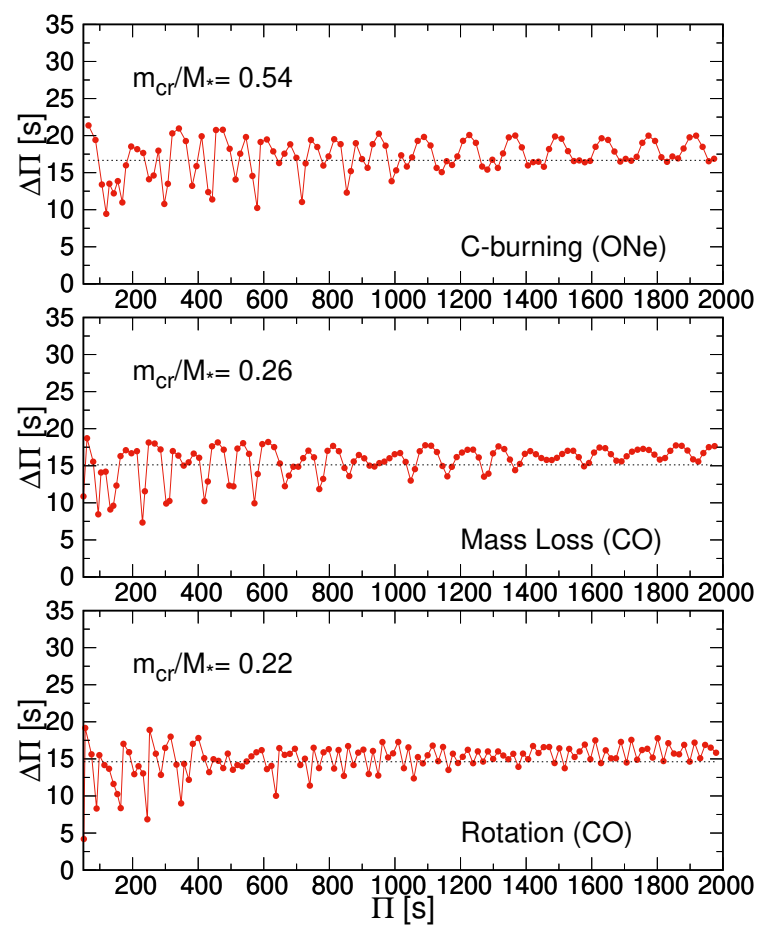

Fig. 8. Same as Fig. 6, but for a stellar mass of $M_{\star}=1.29 M_{\odot}\left(T_{\text {eff }} \sim\right.$ $30000 \mathrm{~K})$.

H-rich objects, but at least a handful of them have been detected in the SDSS (Kleinman et al. 2013; Reindl et al. 2014; Koester et al. 2020; Bédard et al. 2020). In addition, an ultra-massive DB WD with an effective temperature well within the DBV instability strip has been detected by Richer et al. (2019) in a young open cluster, and a hot rapidly rotating DBA WD with a stellar mass of $1.33 M_{\odot}$ has been discovered by Pshirkov et al. (2020). By

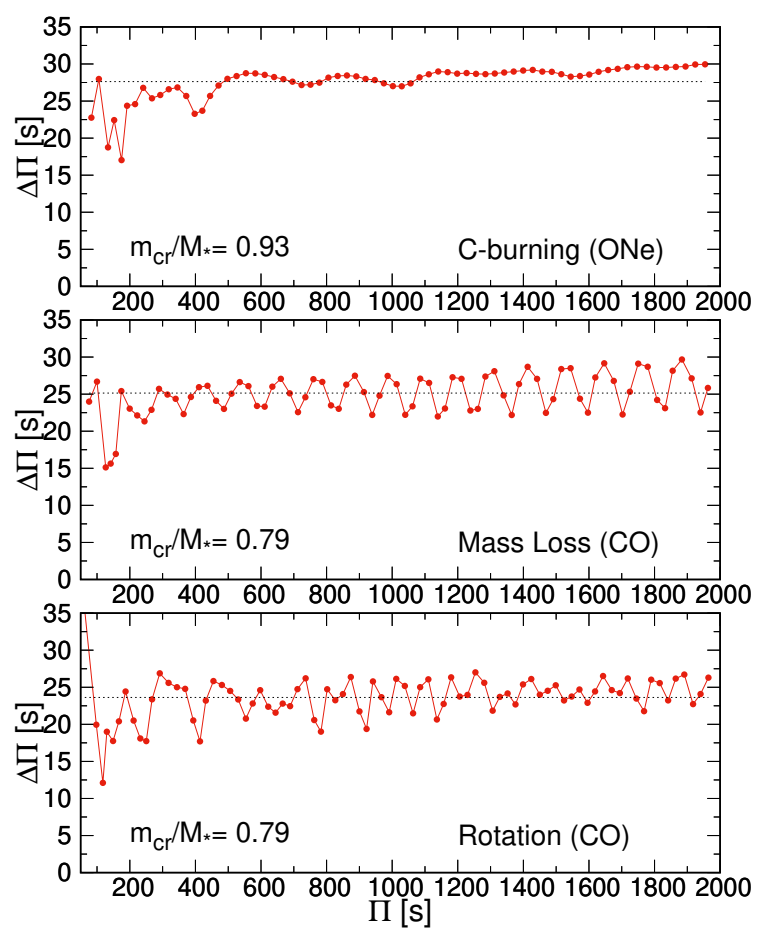

Fig. 9. Same as Fig. 8, but for $T_{\text {eff }} \sim 20000 \mathrm{~K}\left(M_{\star}=1.29 M_{\odot}\right)$.
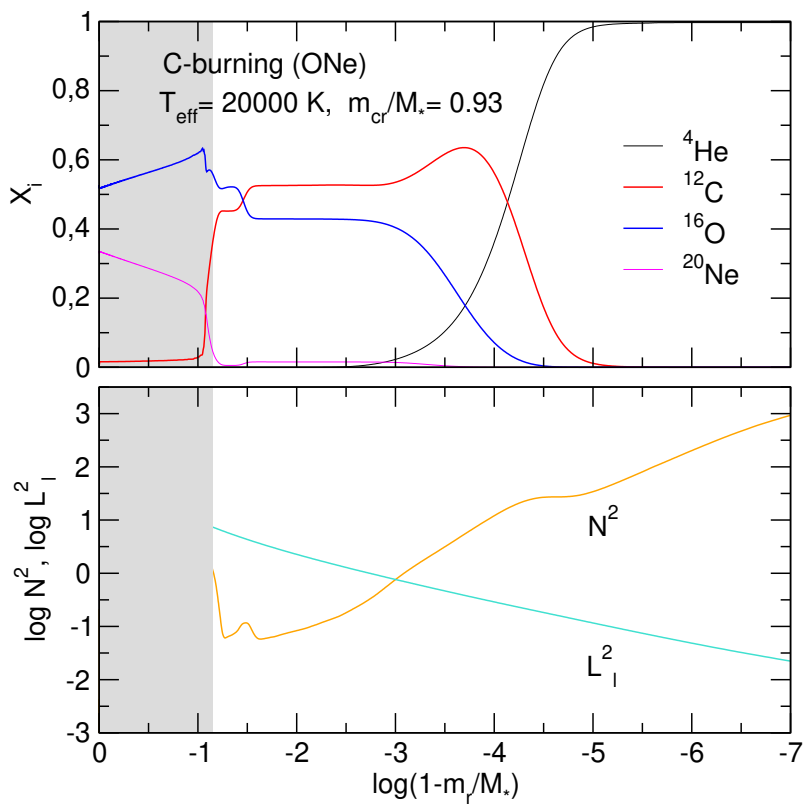

Fig. 10. Upper panel: abundance by mass of ${ }^{4} \mathrm{He},{ }^{12} \mathrm{C},{ }^{16} \mathrm{O}$, and ${ }^{20} \mathrm{Ne}$ versus the outer mass coordinate for the ONe-core $1.29 M_{\odot} \mathrm{DB} W D$ model at $T_{\text {eff }} \sim 20000 \mathrm{~K}$. Lower panel: logarithm of the squared BruntVäisälä and Lamb frequencies $(\ell=1)$.

means of the analysis of the period-spacing and mode-trapping features, we have shown that the pulsational properties of the ultra-massive DB WDs are much more strongly dependent on their formation scenario than in the case of the DA WD ones studied in Althaus et al. (2021). This is due to the fact that DBV stars are much hotter than DAV stars and, therefore, their cores are substantially less crystallized. As a result, $g$-mode pulsations in ultra-massive DBVs can penetrate much deeper into the 
star, thus carrying valuable information about the core chemical structure and composition.

In particular, we expect that pulsating UMCO DB WDs at the hot boundary of the DBV instability strip $\left(T_{\mathrm{eff}} \sim 30000 \mathrm{~K}\right)$ display mode-trapping features with larger amplitudes than those of their ONe-core counterparts (Fig. 6). In the case of very massive pulsating DB WDs $\left(M_{\star} \gtrsim 1.30 M_{\odot}\right)$, we find that if the stars are located near the blue edge of the DBV instability domain, then it would be possible to differentiate between the $\mathrm{CO}$-core of a WD coming from the rotation scenario from the CO-core of a WD coming from the mass-loss scenario and the $\mathrm{ONe}$-core of a WD resulting from the C-burning scenario (Fig. 8). Admittedly, in order to make such a distinction, it would be necessary for the star to show many periods with consecutive radial orders. On the other hand, if the very massive DB WDs are detected near the cool edge of the DBV instability strip ( $T_{\text {eff }} \sim 20000 \mathrm{~K}$, see Fig. 9), then we could have two different situations. If the stars exhibit substantial mode-trapping features, this might reflect that they are ultra-massive CO-core WDs. On the contrary, the absence of mode-trapping signatures would be indicative that the stars have $\mathrm{ONe}$ cores.

We conclude that the eventual detection of pulsating ultramassive DB WDs will constitute a clear and unique opportunity to discern the core composition and origin of the ultra-massive WD population in general. While pulsations have not been detected in any ultra-massive DB WDs so far, there is now an excellent prospect of observing pulsations in WDs in general, and in ultra-massive DB WDs, in particular, thanks to ongoing space missions such as TESS, or space missions that will be operational in the next few years, such as Cheops (Moya et al. 2018) and Plato (Moya et al. 2018).

Acknowledgements. We acknowledge the valuable suggestions and comments of our referee, P.-E. Tremblay, that improved the original version of this paper. Part of this work was supported by PIP 112-200801-00940 Grant from CON ICET, by MINECO grants AYA2014-59084-P, and AYA2017-86274-P, by grant G149 from University of La Plata, and by the AGAUR grant SGR-661/201. This research has made use of NASA Astrophysics Data System.

\section{References}

Althaus, L. G., \& Córsico, A. H. 2004, A\&A, 417, 1115

Althaus, L. G., Serenelli, A. M., Córsico, A. H., \& Montgomery, M. H. 2003, A\&A, 404, 593

Althaus, L. G., Serenelli, A. M., Panei, J. A., et al. 2005, A\&A, 435, 631

Althaus, L. G., Córsico, A. H., Isern, J., \& García-Berro, E. 2010, A\&ARv, 18, 471

Althaus, L. G., Camisassa, M. E., Miller Bertolami, M. M., Córsico, A. H., \& García-Berro, E. 2015, A\&A, 576, A9

Althaus, L. G., Córsico, A. H., Uzundag, M., et al. 2020a, A\&A, 633, A20

Althaus, L. G., Córsico, A. H., \& De Gerónimo, F. 2020b, A\&A, 644, A55

Althaus, L. G., Gil Pons, P., Córsico, A. H., et al. 2021, A\&A, 646, A30

Bauer, E. B., Schwab, J., Bildsten, L., \& Cheng, S. 2020, ApJ, 902, 93

Bédard, A., Bergeron, P., Brassard, P., \& Fontaine, G. 2020, ApJ, 901, 93

Beznogov, M. V., \& Yakovlev, D. G. 2013, Phys. Rev. Lett., 111, 161101

Camisassa, M. E., Althaus, L. G., Córsico, A. H., et al. 2019, A\&A, 625, A87

Castanheira, B. G., Kepler, S. O., Kleinman, S. J., Nitta, A., \& Fraga, L. 2010, MNRAS, 405, 2561

Castanheira, B. G., Kepler, S. O., Kleinman, S. J., Nitta, A., \& Fraga, L. 2013, MNRAS, 430, 50

Chang, P., Bildsten, L., \& Arras, P. 2010, ApJ, 723, 719

Cheng, S., Cummings, J. D., Ménard, B., \& Toonen, S. 2020, ApJ, 891, 160
Christensen-Dalsgaard, J., Silva Aguirre, V., Cassisi, S., et al. 2020, A\&A, 635, A165

Córsico, A. H., \& Althaus, L. G. 2006, A\&A, 454, 863

Córsico, A. H., Althaus, L. G., Benvenuto, O. G., \& Serenelli, A. M. 2001, A\&A, 380, L17

Córsico, A. H., García-Berro, E., Althaus, L. G., \& Isern, J. 2004, A\&A, 427, 923

Córsico, A. H., Althaus, L. G., Montgomery, M. H., García-Berro, E., \& Isern, J. 2005, A\&A, 429, 277

Córsico, A. H., Althaus, L. G., Miller Bertolami, M. M., \& Kepler, S. O. 2019a, A\&ARv., 27, 7

Córsico, A. H., De Gerónimo, F. C., Camisassa, M. E., \& Althaus, L. G. 2019b, A\&A, 632, A119

Curd, B., Gianninas, A., Bell, K. J., et al. 2017, MNRAS, 468, 239

De Gerónimo, F. C., Córsico, A. H., Althaus, L. G., Wachlin, F. C., \& Camisassa, M. E. 2019, A\&A, 621, A100

Decin, L., Homan, W., Danilovich, T., et al. 2019, Nat. Astron., 3, 462

Doherty, C. L., Gil-Pons, P., Siess, L., \& Lattanzio, J. C. 2017, PASA, 34, e056

Dominguez, I., Straniero, O., Tornambe, A., \& Isern, J. 1996, ApJ, 472, 783

Dufour, P., Vornanen, T., Bergeron, P., \& Fontaine, B. A. 2013, in 18th European White Dwarf Workshop, eds. J. Krzesiński, G. Stachowski, P. Moskalik, \& K. Bajan, ASP Conf. Ser., 469, 167

Dunlap, B. H., \& Clemens, J. C. 2015, in 19th European Workshop on White Dwarfs, eds. P. Dufour, P. Bergeron, \& G. Fontaine, ASP Conf. Ser., 493, 547 Fontaine, G., \& Brassard, P. 2008, PASP, 120, 1043

Gagné, J., Fontaine, G., Simon, A., \& Faherty, J. K. 2018, ApJ, 861, L13

Garcia-Berro, E., \& Iben, I. 1994, ApJ, 434, 306

Hermes, J. J., Kepler, S. O., Castanheira, B. G., et al. 2013, ApJ, 771, L2

Hollands, M.A., Tremblay, P.E., Gänsicke, B.T., et al. 2020, Nat. Astron.

Horowitz, C. J., Schneider, A. S., \& Berry, D. K. 2010, Phys. Rev. Lett., 104, 231101

Jiménez-Esteban, F. M., Torres, S., Rebassa-Mansergas, A., et al. 2018, MNRAS, 480, 4505

Kanaan, A., Nitta, A., Winget, D. E., et al. 2005, A\&A, 432, 219

Kepler, S. O., Pelisoli, I., Koester, D., et al. 2016, MNRAS, 455, 3413

Kepler, S. O., Pelisoli, I., Koester, D., et al. 2019, MNRAS, 486, 2169

Kilic, M., Bergeron, P., Kosakowski, A., et al. 2020, ApJ, 898, 84

Kleinman, S. J., Kepler, S. O., Koester, D., et al. 2013, ApJs, 204, 5

Koester, D., Kepler, S. O., \& Irwin, A. W. 2020, A\&A, 635, A103

Maoz, D., Hallakoun, N., \& Badenes, C. 2018, MNRAS, 476, 2584

Medin, Z., \& Cumming, A. 2010, Phys. Rev. E, 81, 036107

Miller Bertolami, M. M. 2016, A\&A, 588, A25

Montgomery, M. H., \& Winget, D. E. 1999, ApJ, 526, 976

Moya, A., Barceló Forteza, S., Bonfanti, A., et al. 2018, A\&A, 620, A203

Pshirkov, M. S., Dodin, A. V., Belinski, A. A., et al. 2020, MNRAS, 499, L21

Reindl, N., Rauch, T., Werner, K., et al. 2014, A\&A, 572, A117

Reindl, N., Schaffenroth, V., Miller Bertolami, M. M., et al. 2020, A\&A, 638, A93

Ricker, G. R., Winn, J. N., Vanderspek, R., et al. 2015, J. Astron. Telesc. Instrum. Syst., 1, 014003

Richer, H. B., Kerr, R., Heyl, J., et al. 2019, ApJ, 880, 75

Rowan, D. M., Tucker, M. A., Shappee, B. J., \& Hermes, J. J. 2019, MNRAS, 486, 4574

Salaris, M., Althaus, L. G., \& García-Berro, E. 2013, A\&A, 555, A96

Schwab, J. 2021, ApJ, 906, 53

Shen, K. J., Bildsten, L., Kasen, D., \& Quataert, E. 2012, ApJ, 748, 35

Siess, L. 2007, A\&A, 476, 893

Siess, L. 2010, A\&A, 512, A10

Silva Aguirre, V., Christensen-Dalsgaard, J., Cassisi, S., et al. 2020, A\&A, 635, A164

Tassoul, M., Fontaine, G., \& Winget, D. E. 1990, ApJS, 72, 335

Temmink, K. D., Toonen, S., Zapartas, E., Justham, S., \& Gänsicke, B. T. 2020, A\&A, 636, A31

Toonen, S., Hollands, M., Gänsicke, B. T., \& Boekholt, T. 2017, A\&A, 602, A16

Tremblay, P. E., Fontaine, G., Freytag, B., et al. 2015, ApJ, 812, 19

Tremblay, P. E., Cukanovaite, E., Gentile Fusillo, N. P., Cunningham, T., \& Hollands, M. A. 2019a, MNRAS, 482, 5222

Tremblay, P.-E., Fontaine, G., Fusillo, N. P. G., et al. 2019b, Nature, 565, 202

Unno, W., Osaki, Y., Ando, H., Saio, H., \& Shibahashi, H. 1989, Nonradial Oscillations of Stars (Tokyo: University of Tokyo Press)

Winget, D. E., \& Kepler, S. O. 2008, ARA\&A, 46, 157

York, D. G., Adelman, J., Anderson, J. E., Jr, et al. 2000, AJ, 120, 1579 